\title{
Design of a Fast and Autonomous Complex Line Tracker and Fully Controlled Robot by Limit Switches
}

\author{
Amevi Acakpovi \\ Accra Polytechnic (Ghana) \\ P.o Box: GP561
}

\author{
Salifu Osman \\ Accra Polytechnic (Ghana) \\ P.o Box: GP561
}

\author{
Olufemi O.Fatonade \\ Accra Polytechnic (Ghana) \\ P.o Box: GP561
}

\begin{abstract}
The explosion of microcontrollers and microprocessor applications has led to a rapid growth in the area of robotics especially line follower robots. Controlling line follower robots to travel over a complex race course pose some instability and speed problems. This article mainly deals with the design of an electronic three wheeled-robot capable of tracking a black line by means of infrared sensors and also capable of moving through a given trajectory by control of limit switches at a relatively high speed. The method consists first in developing a program written in Basic language under Microcode plus environment and loading it into the microcontroller to control the robot. Various PWM techniques were adopted in the programming to handle the turning left, right, the moving forward and backward. In addition, necessary hardware involving 6V DC motor, limit switches, infrared sensors, $6 \mathrm{~V}$ battery and others have been assembled on a PIC16F877 microprocessor board and put in a case, shaped as a vehicle. $\mathrm{H}$ bridge motor control and PWM were used to control the DC motors. The implementation is finally successful. The robot can move autonomously across a black line and also pass through a complex race in absence of black line by means of limit switches. The motor can complete a race of $2 \mathrm{~m}$ in $10 \mathrm{~s}$ and turn at angles less than 30 degree while remaining stable.
\end{abstract}

\section{General Terms}

Automation, Microprocessor/Microcontroller programming, Intelligent wireless sensor programming.

\section{Keywords}

Basic language Programming, Microprocessor, DC motor control, infrared sensors, PWM, H-bridge.

\section{INTRODUCTION}

Line follower robots have played a significant role in industries growth. Initially, used for the purpose of delivering mails within an office building, they are now serving to deliver medication in a hospital. With more accuracy in the design, the technology could even be suggested for running buses and other mass transit systems, and may end up as part of autonomous cars navigating the freeway. Many automation techniques help to design such robots. In the scope of our design the work will be focused on the use of PIC microcontrollers with programming of PWM for the control of the DC motors.

\section{PROBLEM STATEMENT}

Line follower robots are popular in robotics industry. The ultimate reason to design a new robot is to have it computing with others in terms of speed and stability to travel over a complex race course. These factors lead to the following questions: What type of mechanical design can provide both speed and stability? What type of control could be applied on the motors to achieve a higher speed? How will the sensing of black line be done?

\section{OBJECTIVES}

The main objective of this work is to design and implement a fast and autonomous robot that will follow a black line on a white board and also follow a given trajectory by using limit switches in absence of black line.

The following specific objectives will help achieving the main purpose:

- Design of a three wheels mechanically balanced robot

Design of various PWM applicable for the DC motors using Basic language, in order to achieve turning right, turning left, moving forward or moving backward of the motor.

Testing of the motor over a complex course race and adjusting the PWM to achieve the fastest speed

\section{REVIEW OF LITERATURE}

Extensive research works were done on the mechanical aspect using different methods to get robots moving fast. Some include tank tread robot, two wheels robot, omnidirectional four wheel robot, six wheel robots. The tank tread robot, developed by Boe-Bot [1] has a strong mechanical aspect which allows it to adapt itself to any varied terrain such as carpet, small rocks, imperfect surfaces but it does not achieve any high speed. However the omnidirectional four wheels designed by L.Huang et al [2] show superiority to those with differential wheels in terms of dexterity and driving ability. Their controller is made of an RF unit which receives the command and forwards it to the microcontroller. The microcontroller then generates the PWM pulses and sends them to the appropriate motors through their driver. These robots are capable of performing various difficult movements. Results were shown in term of travelling distance and speed control of the same robot. The robot traces a line approximately straight which is acceptable at a speed that increases gradually from 0 to a threshold of $1000 \mathrm{~mm} / \mathrm{s}$. 
Once again, the four-wheel model is not initially based on the speed aspect but rather on the stability and mobility. However additional works done respectively by Pedro F. Santana et al [3], Dmitry U. Panfilov et al [4], Thomas Bak et al [5] and Christophe Cariou et al [6] try to improve upon the speed of wheeled motors. Furthermore, several other types of control apply to wheeled robot: Gyula Mester [7] uses kinematic controls on a two wheeled robot with control strategies using a feed-forward compensator. Other motion controls by Thomas Bak et al [5] and Sonal Kalra et al [8] were totally hybrid type. They mainly use a non-holonomic dynamic system to model the robot. Finally Rick Bickle [9] and Electronics Design Laboratory [10] designed two fundamental control methods to apply to the DC motors to achieve high speed: $\mathrm{H}$ bridge method and PWM. The H-bridge enables the voltage to be applied in both directions allowing both forward and backward movement. The PWM has direct effect on the speed.

\section{METHODOLOGY}

This project involves both hardware and software aspects. The design description will first start with the hardware aspect and explain their configuration after which will follow the software description.

\subsection{Hardware Design}

The hardware deals with the mechanical design of the robot. The robot is made of four fundamental parts: Four limit switches coupled with bumpers, three infrared sensors used to track black line, the main microprocessor PIC16F877 board with LCD screen, the two DC-motor, their driving circuit and a $6 \mathrm{~V}$ battery with charger. Two tyres are positioned at each sides at the back and the third on is in front. The front tyre is not coupled with any motor but can rotate in any direction. Figure 1 shows the bloc diagram of the robot controller. The shape of the robot is illustrated by Figure 2 .

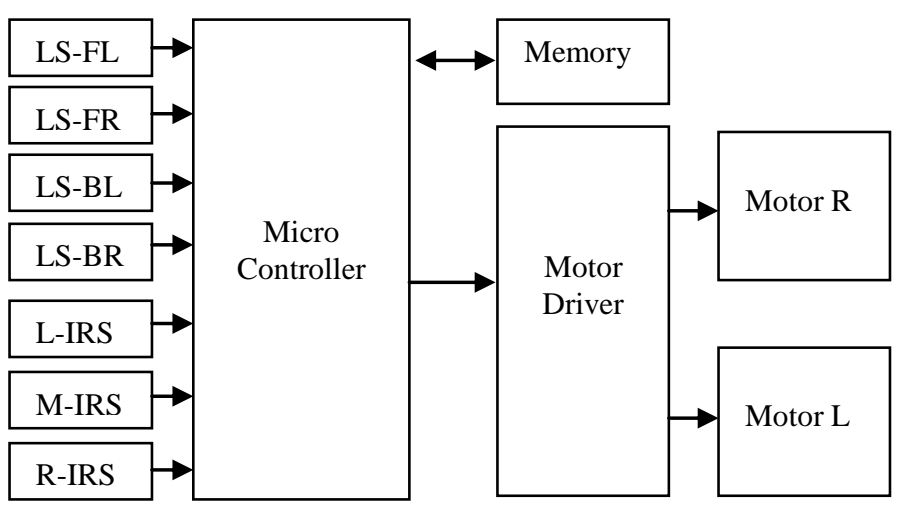

Figure 1: Block diagram of the robot controller

Legend:

LS-FL: Limit switch front left

LS-FR: Limit switch front right

LS-BL: Limit switch back left

LS-BR: Limit switch back right

Motor R: DC Motor right side

Motor L: DC Motor left side

Limit switches
The limit switches are put in line under the car, two at the front and two at the back of the robot. In between the switches in front are two bumpers to enable the car detect obstacles. Figure 3 shows a picture of the configuration of the limit switches. When the bumper hit an obstacle, it closes the corresponding switch which sends a signal to the microprocessor. The signal is interpreted to take decision base on the program that is further explained.

\section{Infrared sensors}

They consist of a set of three infrared sensors put together on the same printed circuit board and centered under the base board of the robot. The spacing between two consecutive sensors is about $1 \mathrm{~cm}$. Each sensor comprises both an infrared transmitter and receiver. The transmitter emits an invisible radiation which is sensed back by the receiver in case it falls on any object having black color. A solution-tape of $2 \mathrm{~cm}$ width is set on a white board and the sensors are supposed to sense them and make the car follow the black line.

\section{Main microprocessor board}

The main board is made of the PIC16F877 microprocessor with adequate extension to the various input and output ports and also an LCD screen.

\section{LCD Screen}

The LCD (liquid crystal display) screen is a digital screen capable of printing 20 digits, including alphanumerical characters. It is used in this project to display information on the identity of the car. For instance the text displayed on the LCD screen is: "Robot PIC16F877.

\section{DC motors}

Two 6V DC motor were used to control the two tyres at the back independently. The motors can be controlled to move in both directions using a drive circuit that will be further explained.

\section{Battery pack}

The whole design is powered with a $6 \mathrm{~V}$ rechargeable battery. The battery capacity of 12 Ah allow the robot to move continuously for 2 hours.

\section{Motor driving circuit}

An $\mathrm{H}$ bridge DC motor direction control has been used with complemented inputs as shown by the Figure 4 . The driver circuit is built for each of the DC motor. The three pins of connector $\mathrm{J} 2$ are made available for connection to the microprocessor board. The three pins on connector $\mathrm{J} 2$ are VCC, Control and GND. When the control signal sent from the microcontroller is digital 1, Q2 and Q4 are forward biased and then run the motor in Forward Direction. Conversely, when the control voltage comes to a 0 logic, Q1 and Q3 become forward biased and run the motor in reverse direction.

\subsection{Software Description}

The software description involves the use of artificial intelligence to execute the movement of the car. This is based on a specific program written in basic language and explained by the flowchart in Figure 5. The three infrared sensors are considered digital inputs. The 0 level means the sensor has detected a black line. All the decision boxes in the flowcharts are checking if the sensors have detected the black line or not. 
At start, the robot goes through an initialization process which involves the definition of input and output ports after which it beeps for 2 seconds and display a message on the LCD screen. The program continues by checking the state of "BITSART" which is a push button that the user must press to run the program. At the execution of the main program, the robot move in one of the following direction according to the state of black line sensed by the three infrared sensors: forward, backward, left, right, normal left or normal right.

\section{Forward}

The forward procedure is explained by the flowchart in Figure 6. First the program checks if the back left or right switches are hitting an obstacle in which case it execute different procedures called "EVADE_LEFT" and "EVADE_RIGHT respectively. If there is no obstacle detected, the program runs the left and the right motors for $2 \mathrm{~ms}$ and $1 \mathrm{~ms}$ respectively, insert a delay of $18 \mathrm{~ms}$ and return back to the main program to check if the state of the infrared sensors continues to allow the robot to move forward.

\section{Evade Left}

This procedure allows the robot to evade left, which is explained in Figure 7. It is executed when the front left switch hit an obstacle. It is assumed to help the robot to change direction and avoid the obstacle. It consists of moving the robot backward for a certain distance and turns it right for $45^{\circ}$. Otherwise, while moving backwards if the left or right back switches detect obstacles the robot turn right or left respectively at an angle of $45^{\circ}$.

\section{Evade Right}

The procedure "EVADE_RIGHT" does the same thing as the "EVADE_LEFT" just that the process starts based on the detection of the front right switch. In this case the robot moves backward and turn left.

\section{Turn Right}

As shown by the Figure 8, the robot turns right when there is no detection of black line. It turns right continuously, resulting into rotation until the sensors meet a black line. The procedure consists of turning on and off consecutively the right side motor and the left side motor for $2 \mathrm{~ms}$ each and inserting a delay of $18 \mathrm{~ms}$. A click on the push button start can stop this procedure and go back to the main program. This is to allow the user to relocate the robot in order to detect a black race course instead of turning right continuously.

\section{Turn Left}

This procedure is similar to the previous one but the whole system turns left.

\section{Turn Normal Right}

It is a slow turning into the right direction which is often called several times to allow the motor to turn at a desired angle. The procedure consists of turning the left motor for $2 \mathrm{~ms}$ while keeping the right one off and then inserting $18 \mathrm{~ms}$ delay. Figure

9 shows the flowchart.

\section{Turn Normal Left}

This procedure does the same thing as the "TURN NORMAL RIGHT" just that the turning direction is left.

\section{Backwards}

Backward is achieved by turning on sequentially, the left motor for $1 \mathrm{~ms}$, the right one for $2 \mathrm{~ms}$ and inserting a pause of $18 \mathrm{~ms}$. Figure 10 illustrate it.

\section{DISCUSSION OF FINDINGS}

The completed robot is very stable owing to the mechanical aspect of the design. The robot was tested several times over a complex course race. It can travel over a straight line of 2 meters within $10 \mathrm{~s}$. The robot can also turn left or right at angles less than 30 degree without turning over, showing a considerable stability. In absence of black line the robot movement is controlled by the limit switches. For instance, if any of the front switches hit an obstacle, the robot move backward and turn slightly in the opposite direction before moving forward. This helps the robot to overpass an obstacle after hitting it for a certain limited number of times. This number is not fixe but depends upon the angle at which the robot hit the obstacle for the first time.

Furthermore, the timing diagram of Figure 11 explains the PWM applied for the forward and backward procedures. This diagram is derived from the flowcharts explained above. The average voltage level for both motors is given by:

$$
V_{a v}=\frac{1}{T} \int_{0}^{T} v(t) d t
$$

After applying the formula, the voltage levels are respectively, $57 \%$ and $29 \%$ for the Left and Right motors.

\section{CONCLUSION}

This article presents the design of a robot capable of following a black line and also capable to move in absence of black line with the control of four limit switches. The robot is successfully designed and capable of passing through medium to complex races at a relatively high speed. The robot is controlled by a program written in Basic language and loaded on a PIC16F877 controller which involves different PWM to control the various movements (turning left, turning right, moving forward or backward...). The design is an applied engineering example of using microprocessor/microcontroller in Electricity, Electronics and Computer technology.

Further works could improve the robot design by considering a more advanced mechanical shape (4 wheels) and also by using a speed drive for the DC motors in order to increase their speed.

\section{ACKNOWLEDGMENTS}

Our thanks go to the experts who have contributed to the development of this line tracker robot, especially: Nicholas Ankrah and Samuel Inkumsa.

\section{REFERENCES}

[1] Boe-Bot 2011. Boe-Bot Tank Treads. www.parallax.com.

[2] Huang,L., Lim, Y.S., David Li and Christopher, E.L. 2004. Design and Analysis of a Four-wheel Omnidirectional Mobile Robot. $2^{\text {nd }}$ International Conference on Autonomous Robot and Agents, Decenber 13-15 2004, Palmerstorn North, New Zeland.

[3] Pedro, F. S., Carlos C. and Vasco S. 2006. A Motion Controller for Compliant Four-Wheeled-Steering Robots. IntRoSys, S.A., FCT-UNL 2829-516- Portugal. 
[4] Dmitry U. P. and Sergey B. T. 2007. Tracking of reference trajectory for wheeled mobile robot. Bauman Moscow State Technical University, ul. 2-aja Baumanskaja, 5, 107005, Russia., Moscow.

[5] Thomas B., Jan B. and Anders P. R. 2011. Hybrid Control Design for a Wheeled Mobile Robot. Department of Control Engineering, Aalborg University, Fredrik Bajers Vej 7C, DK-9220, Aalborg, Denmark.

[6] Christophe C., Roland L., Benoit T. and Michel B. 2009. Automatic Guidance of a Four-Wheel-Steering Mobile Robot for Accurate Field Operations. Journal of Field Robotics 26(6-7), 504-518 (2009), Wiley InterScience.
[7] Gyula M. 2006. Motion Control of Wheeled Mobile Robots. SISY. $4^{\text {th }}$ Serbian-Hungarian Joint Symposium on Intelligent System.

[8] Sonal K., Dipesh P. and Karl S. 2007. Design and Hybrid Control of a Two Wheeled Robotic Platform. Dept. of Mechanical Engineering, University of Auckland, New Zealand

[9] Rick.B. 2003. DC Motor Control Systems For Robot Applications.

[10] Electronics Design Laboratory. 2003. Robot DC Motor Driver and Speed Control. ECEN 2830

\section{APPENDIX I: FIGURES}

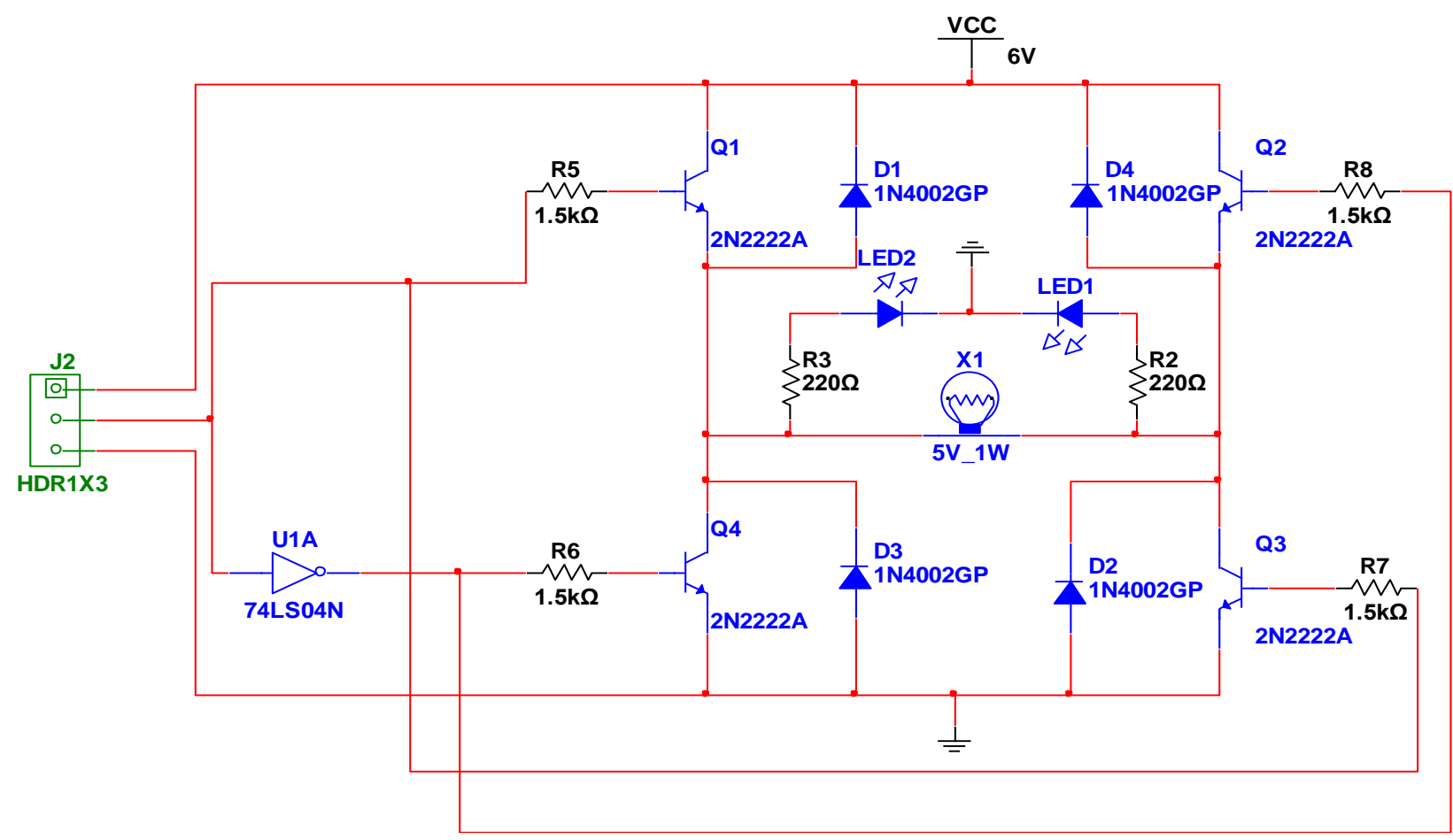

Figure 4: H Bridge Driver Circuit 


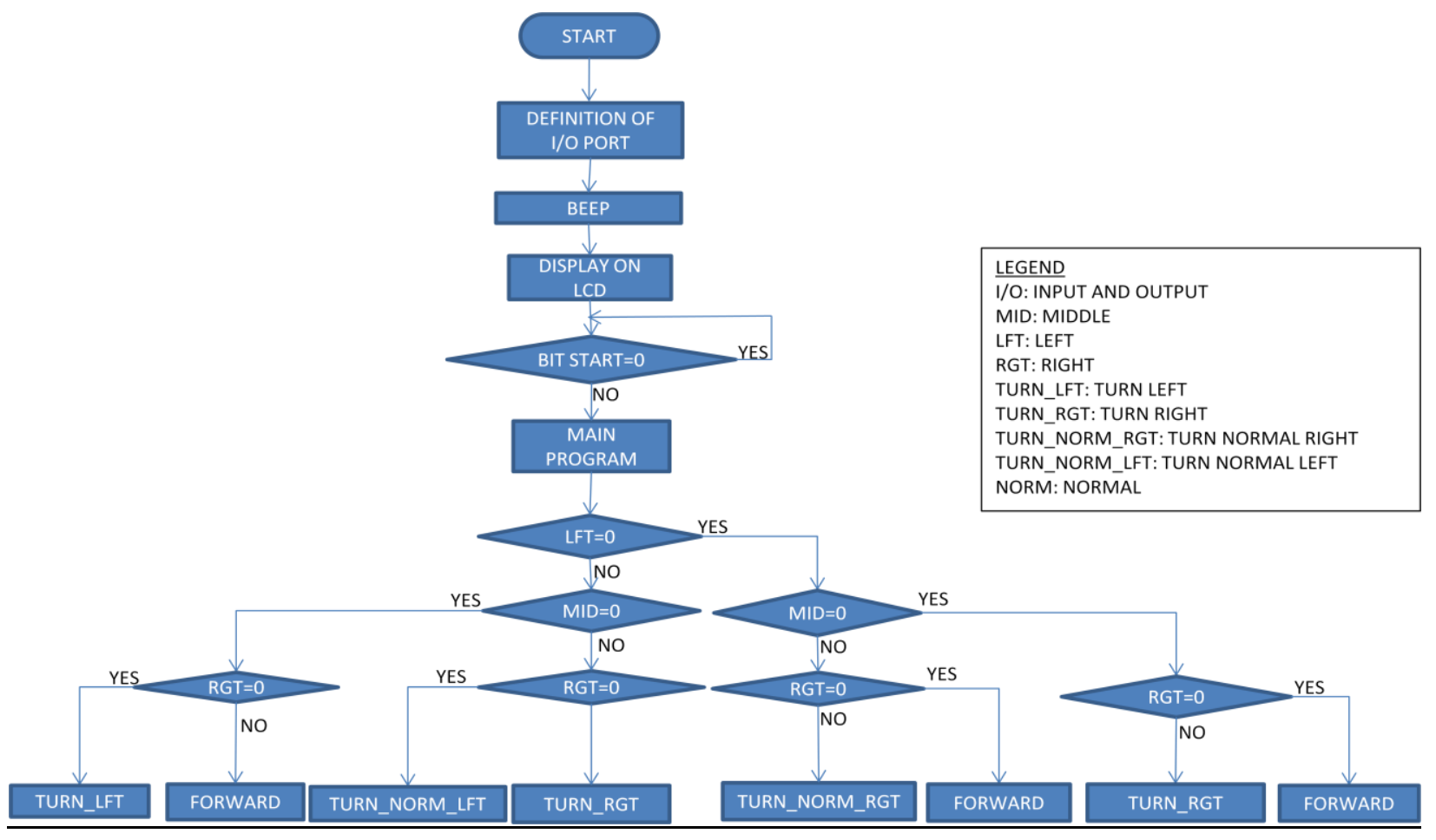

Figure 5: Main program

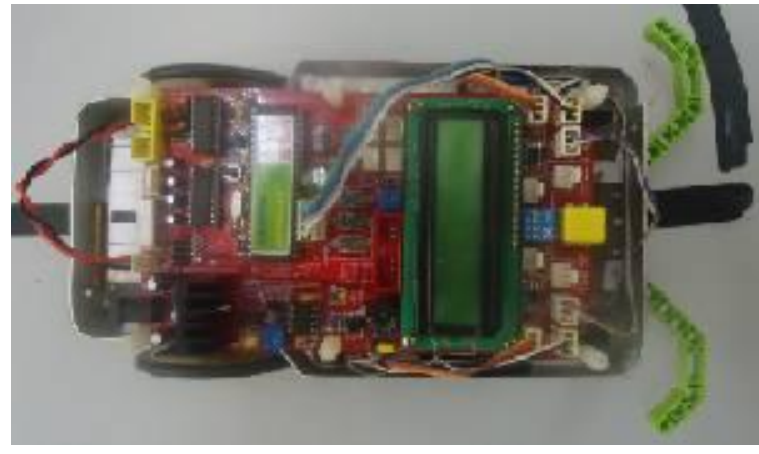

Figure 2: Three-wheeled robot

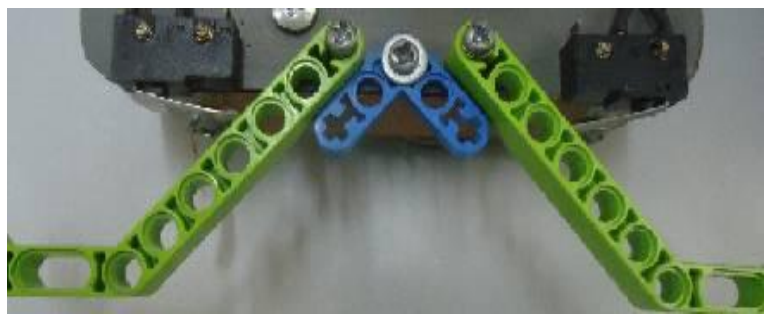

Figure 3: Infrared sensor

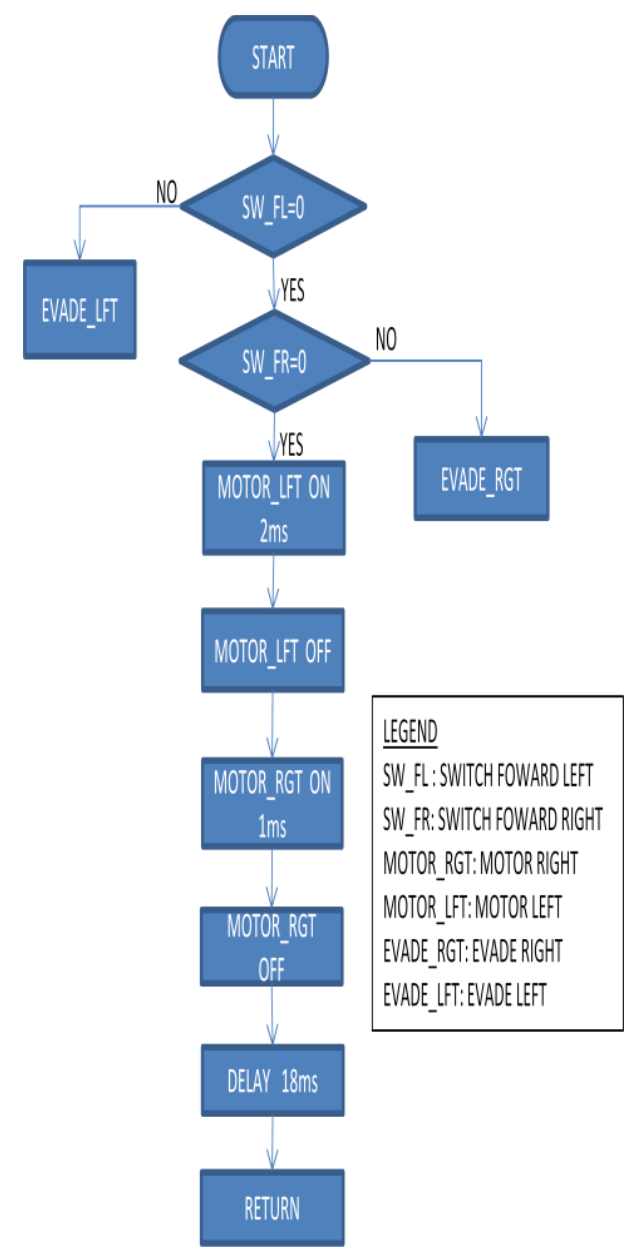

Figure 6: Forward 

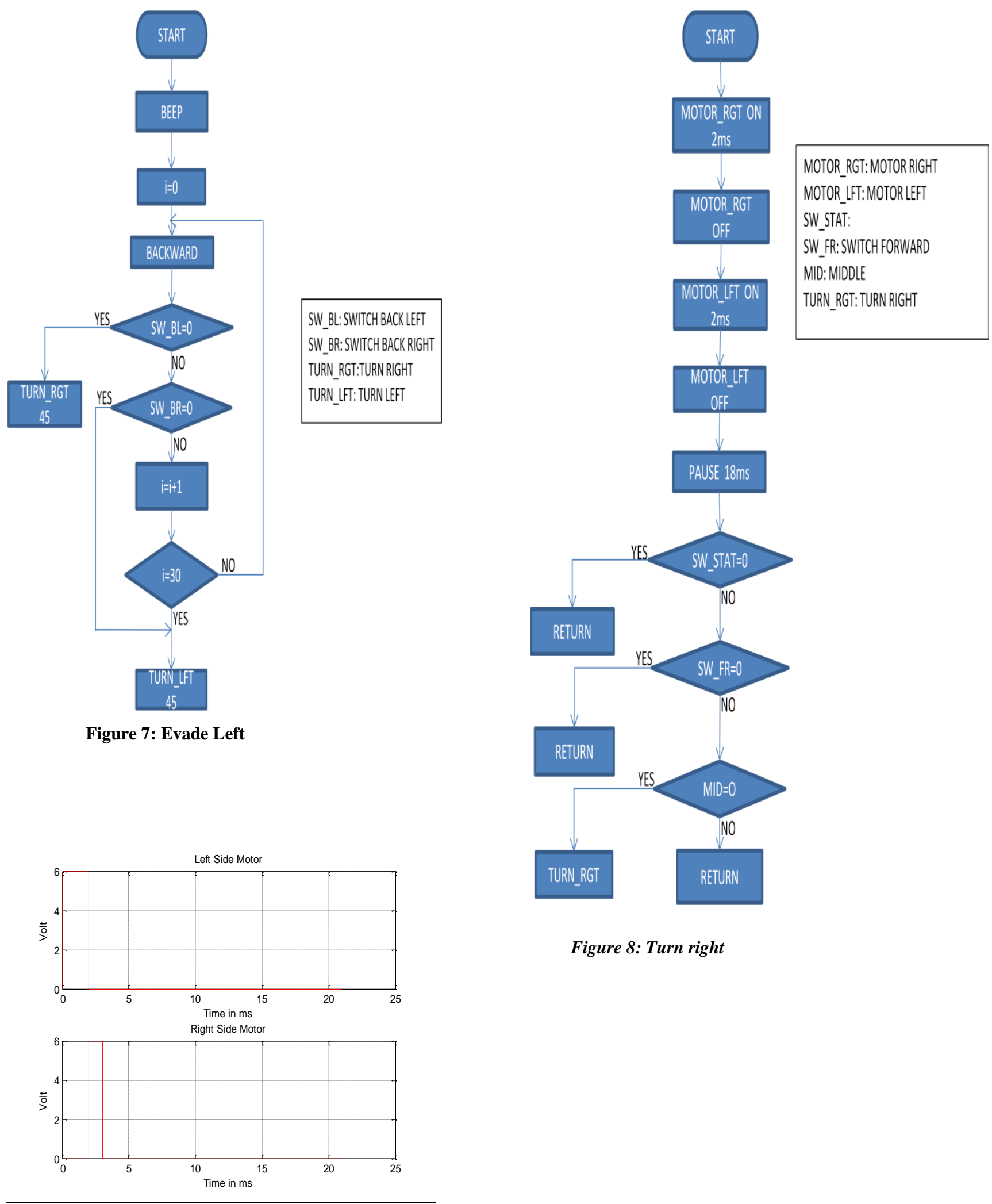

Figure 8: Turn right

Figure 11: PWM waveform for Backward and Forward procedures 

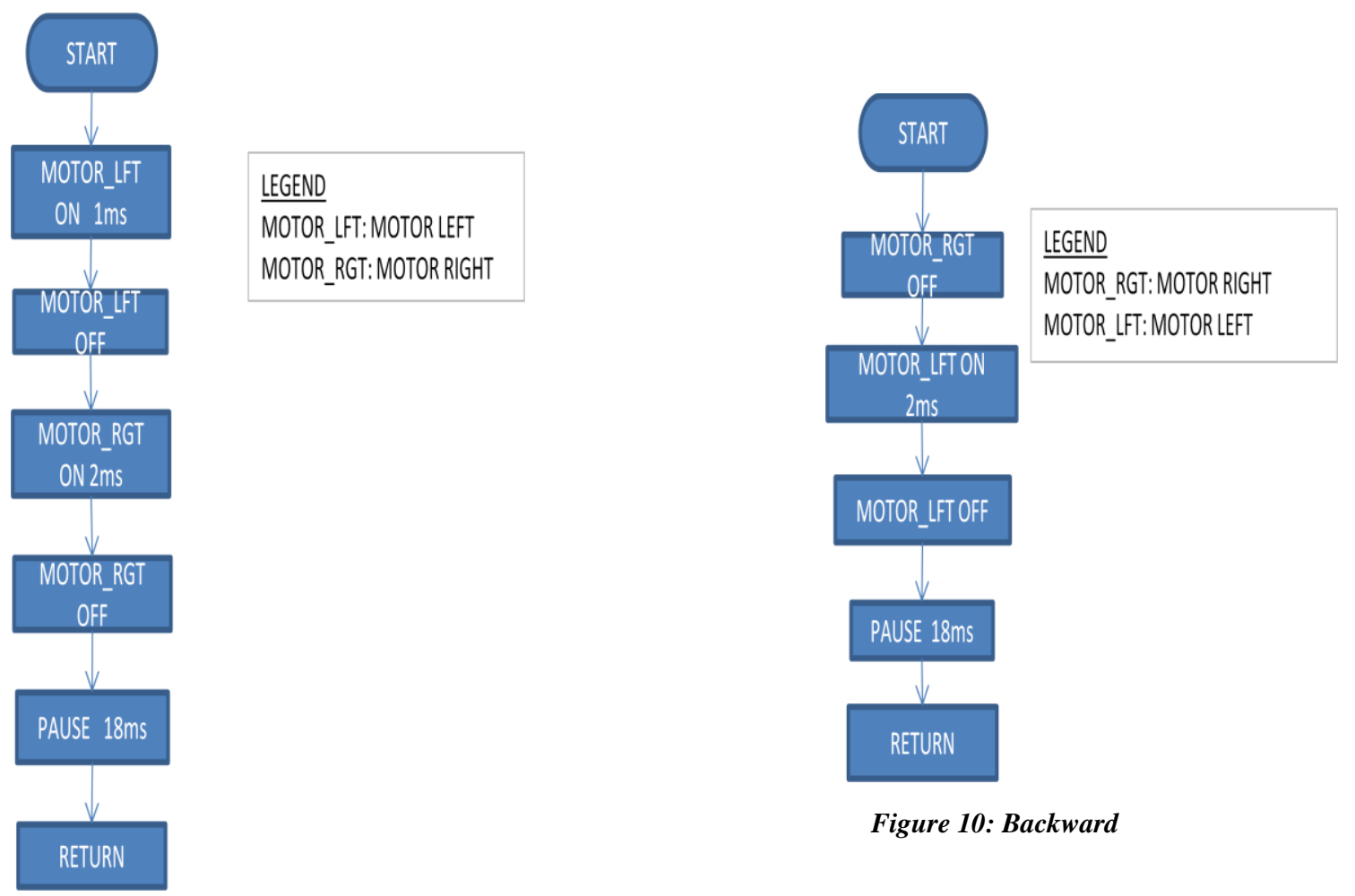

Figure 10: Backward

Figure 9: Turn normal right

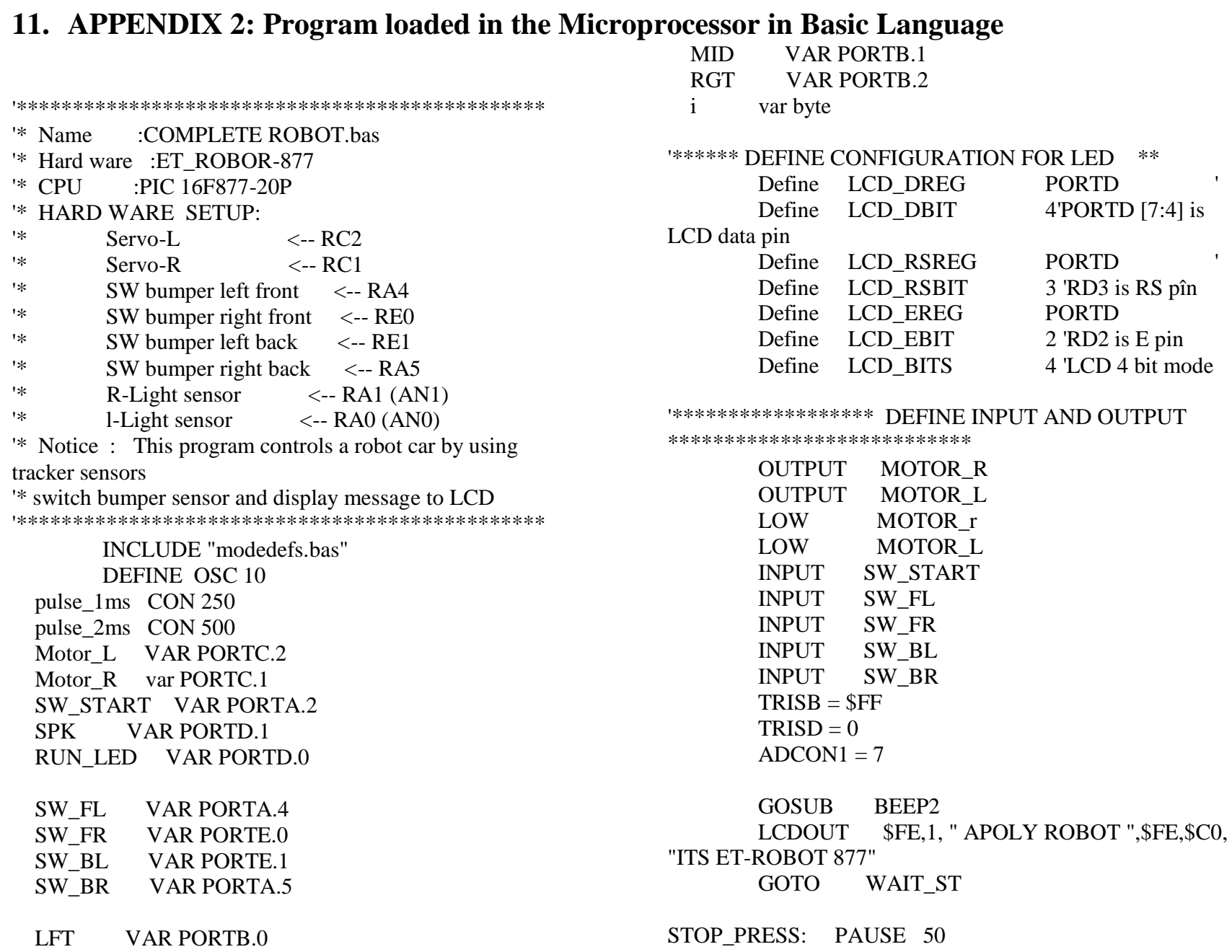


